Proceeding Series of the Brazilian Society of Computational and Applied Mathematics

\title{
Conjuntos Compactos: Definição e Exemplos
}

\section{Livea Cichito Esteves}

Faculdade de Engenharia de Ilha Solteira, Unesp, Ilha Solteira, SP

\section{Luis Antonio Fernandes Oliveira ${ }^{2}$}

Departamento de Matemática, Unesp, Ilha Solteira, SP

Resumo. Neste trabalho, estudaremos os conjuntos compactos. Vamos definir cobertura, cobertura aberta, subcobertura e subcobertura aberta, para então ser possível definirmos os conjuntos compactos. Em seguida, veremos alguns exemplos e propriedades que este tipo de conjunto possui.

Palavras-chave. Topologia, Compacidade, Cobertura, Subcobertura.

\section{Introdução}

A teoria de Conjuntos Compactos é de grande importância na Matemática, pois possibilitou que Teoremas clássicos de Análise Real ganhassem versões em um contexto mais geral. Dentre as contribuições obtidas, o conceito de compacidade, propiciou o entendimento de certos fatos válidos para intervalos fechados e limitados na reta real e peculiaridades dos conjuntos compactos no âmbito dos espaços métricos e topológicos.

Em Topologia, o conceito de compacidade é uma extensão topológica das ideias de finitude e limitação.

Definição 1.1: Seja $(E, \boldsymbol{\tau})$ um espaço topológico e $X$ um subconjunto de $E$. Uma cobertura de $X$ é uma coleção $A=\left(A_{\lambda}\right)_{\lambda \in L}$ de subconjuntos do espaço topológico $E$, tal que $X \subset \mathrm{U}_{\lambda \in \mathrm{L}} A_{\lambda}$.

Uma cobertura é dita aberta se os elementos de $A$ são abertos em $E$ :

$$
\left(A_{\lambda}, \lambda \in \gamma ; X \subset U_{\lambda \in \gamma} A_{\lambda}\right)
$$

Definição 1.2: Seja $A=\left(A_{\lambda}\right)_{\lambda \in L}$ uma cobertura de $X$. Uma subcobertura de $A$ é uma subcoleção $A^{\prime}=\left(A_{\lambda^{\prime}}\right)_{\lambda^{\prime} \in L^{\prime}}, L^{\prime} \subset L$, que ainda é uma cobertura de $X$, isto é, $X \subset \cup_{\lambda^{\prime} \in L^{\prime}} A_{\lambda^{\prime}}$. [2-3]

Uma subcobertura é finita se a quantidade de subconjuntos é finita.

${ }^{1}$ livea_unica@hotmail.com

2 lafo@mat.feis.unesp.br 
Definição 1.3: Um subconjunto $K$ de um espaço topológico $E$ é dito compacto quando toda cobertura aberta de $K$ admitir uma subcobertura finita. [3]

Dessa forma, se quisermos mostrar que um conjunto $X$ não é compacto basta encontrar uma cobertura aberta de $X$ que não admita subcobertura finita. Na reta real, um conjunto é compacto se e somente se, é fechado e limitado (ver Teorema de BorelLebesgue).

\section{Exemplos}

1. A reta $\mathbb{R}$ não é um conjunto compacto. Consideremos a cobertura aberta $A=\left(A_{n}\right)_{n \in \mathrm{N}}$, tal que $A_{n}=(-n, n)$. Essa cobertura aberta não admite subcobertura finita, pois a união de um número finito de intervalos $(-n, n)$ é igual ao intervalo de maior índice, que nunca será igual a $\mathbb{R}$, e dessa forma $\mathbb{R}$ não estará contido nessa união. [1]

2. Se $E$ é um espaço topológico qualquer, então cada subconjunto finito de $E$ é compacto.

3. Se $E$ é um espaço topológico finito, então todo subconjunto de $E$ é compacto.

4. Se $K, L$ são subconjuntos compactos de um espaço topológico $E$, então $K \cup L$ é compacto.

5. Todo subconjunto compacto $K$ de um espaço métrico $M$ é necessariamente limitado.

6. Seja $E$ um espaço de Hausdorff. Todo subconjunto compacto $K \subset E$ é fechado em $E$.

7. A imagem de um conjunto compacto por uma aplicação contínua é um conjunto compacto. [3]

\section{Agradecimentos}

Agradeço ao professor Luis Antonio Fernandes de Oliveira pela orientação neste trabalho e ao CMAC pela oportunidade de participar deste Congresso.

\section{Referências}

[1] S. M. da Luz, Conjuntos Compactos, Trabalho de Conclusão de Curso de Matemática, Universidade Federal de Santa Catarina, (2000).

[2] J. A. E. Dieudonné, Foundations of modern analysis, London, Academic Press, (1969).

[3] E. L. Lima, Elementos de Topologia Geral, Rio de Janeiro, Ao Livro Técnico S/A, (1969). 